\title{
Association between C-reactive protein and radiotherapy-related pain in a tri-racial/ ethnic population of breast cancer patients: a prospective cohort study
}

\author{
Eunkyung Lee ${ }^{1 *}$ D, Omar L. Nelson², Carolina Puyana ${ }^{2}$, Cristiane Takita ${ }^{3}$, Jean L. Wright ${ }^{4}$, Wei Zhao ${ }^{5}$,
} Isildinha M. Reis ${ }^{2,5}$, Rick Y. Lin², WayWay M. Hlaing ${ }^{2}$, Johnna L. Bakalar ${ }^{2}$, George R. Yang ${ }^{2}$ and Jennifer J. Hu ${ }^{2,5^{*}}$

\begin{abstract}
Background: Post-surgery adjuvant radiotherapy (RT) significantly improves clinical outcomes in breast cancer patients; however, some patients develop cancer or treatment-related pain that negatively impacts quality of life. This study examined an inflammatory biomarker, C-reactive protein (CRP), in RT-related pain in breast cancer.

Methods: During 2008 and 2014, breast cancer patients who underwent RT were prospectively evaluated for preand post-RT pain. Pre- and post-RT plasma CRP levels were measured using a highly sensitive CRP ELISA kit. Pain score was assessed as the mean of four pain severity items (i.e., pain at its worst, least, average, and now) from the Brief Pain Inventory. Pain scores of 4-10 were classified as clinically relevant pain. Multivariable logistic regression analyses were applied to ascertain the associations between CRP and RT-related pain.

Results: In 366 breast cancer patients (235 Hispanic whites, 73 black/African Americans, and 58 non-Hispanic whites), $17 \%$ and $30 \%$ of patients reported pre- and post-RT pain, while $23 \%$ of patients had RT-related pain. Both pre- and post-RT pain scores differed significantly by race/ethnicity. In multivariable logistic regression analysis, RTrelated pain was significantly associated with elevated pre-RT CRP ( $\geq 10 \mathrm{mg} / \mathrm{L}$ ) alone (odds ratio $(O R)=2.44 ; 95 \%$ confidence interval $(C l)=1.02,5.85)$; or combined with obesity $(O R=4.73 ; 95 \% \mathrm{Cl}=1.41,15.81)$ after adjustment for age and race/ethnicity.
\end{abstract}

Conclusions: This is the first pilot study of CRP in RT-related pain, particularly in obese breast cancer patients. Future larger studies are warranted to validate our findings and help guide RT decision-making processes and targeted interventions.

Keywords: Breast cancer, Radiotherapy, Pain, C-reactive protein, Inflammatory biomarker

\section{Background}

Breast cancer is the most frequently diagnosed cancer and the second leading cause of cancer death among American women [1]. Compared to breast-conserving surgery (BCS) alone, adjuvant radiotherapy (RT) has significantly reduced loco-regional recurrences [2]. However, RT-induced adverse responses negatively impact

\footnotetext{
* Correspondence: eunkyung.lee@ucf.edu; jhu@med.miami.edu

'Department of Health Sciences, University of Central Florida College of Health Professions and Sciences, Orlando, FL 32816, USA

${ }^{2}$ Department of Public Health Sciences, University of Miami School of Medicine, Miami, FL 33136, USA

Full list of author information is available at the end of the article
}

patient overall quality of life (QOL). Breast erythema, pain, retraction at the tumor-bed site, fibrosis, cardiac morbidity, lymphedema, and telangiectasia are among the known adverse responses to RT [3-6]. Pain is one of the most prevalent symptoms and is an important QOL issue in breast cancer survivors [7-10].

A recent study reported the presence of racial-ethnic disparities in pain experience upon completion of RT [11], indicating the heterogeneity in the RT responses. The identification of a biomarker that can predict treatment-related symptoms is an important research question in the field of radiation oncology. Exposure to

(c) The Author(s). 2019 Open Access This article is distributed under the terms of the Creative Commons Attribution 4.0 International License (http://creativecommons.org/licenses/by/4.0/), which permits unrestricted use, distribution, and reproduction in any medium, provided you give appropriate credit to the original author(s) and the source, provide a link to the Creative Commons license, and indicate if changes were made. The Creative Commons Public Domain Dedication waiver (http://creativecommons.org/publicdomain/zero/1.0/) applies to the data made available in this article, unless otherwise stated. 
ionizing radiation induces immune/inflammatory responses to promote tissue repair [12], and elevated proinflammatory cytokines are potential biomarkers for RT-induced toxicities [13-15]. However, very few studies have examined biomarkers for RT-related pain. Recently, our lab reported that RT-induced skin toxicity was associated with an increase in plasma $\mathrm{C}$-reactive protein (CRP) levels $[15,16]$. This may suggest a potential relationship between inflammatory responses and RT-induced skin toxicities, which can be another source of treatment-related pain for patients with breast cancer.

CRP has been widely used as a robust inflammatory biomarker for many health conditions in both clinical and research settings, and several studies have shown a positive correlation between plasma CRP levels and pain intensity in cancer patients [17-19]; however, these results were from cross-sectional studies, which were often limited by uncertain temporal relationships or from the univariate analysis without adjustment for confounding variables [20, 21]. In addition, the study samples were limited to a specific racial/ethnic group, resulting in limited generalizability of the findings.

Therefore, we aimed to examine the associations between CRP levels and RT-related pain among breast cancer patients who underwent adjuvant RT using a prospective study design. We hypothesized that breast cancer patients with elevated CRP levels would be more likely to report pain, which may identify CRP as an inflammatory biomarker for pain. We also hypothesized that patients with elevated pre-RT CRP may be at higher risk in developing RT-related pain. Pain sensitization is one of the most important risk factors for persistent pain [22, 23]; thus, identifying potential biomarkers or mediators will be a critical strategy to identify those at risk of RT-related pain and targeted interventions among breast cancer patients.

\section{Methods}

\section{Study design and patient population}

Data for the current analysis was obtained from a prospective cohort study (University of Miami, FL, USA) where the goal was to examine the disparity of RT-induced early adverse skin reactions in a racially and ethnically diverse population of breast cancer patients. Briefly, the study recruited breast cancer patients from the Radiation Oncology clinics at the University of Miami Sylvester Comprehensive Cancer Center and Jackson Memorial Hospital in Miami, Florida, between December 2008 and August 2014. Patients were followed up for up to 12 months after the completion of RT. At the time of enrollment, each participant completed a self-administered baseline questionnaire. In addition, participants completed QOL questionnaire on the first day before initiation of RT, on the last day immediately after completion of RT, and at each follow-up visit $(1,2,6$, and 12 months). The current study only used QOL data collected on the first day of RT (i.e., pre-RT) and on the last day of RT (i.e. post-RT). The treating radiation oncologist met patients each week during the radiation treatment and evaluated adverse skin reactions at week 3 (mid-treatment), at week 6 (completion of RT), and at each follow-up visit. We collected blood samples $(20 \mathrm{~mL})$ at pre- and post-RT for biomarker data. Blood samples were processed within 2 $\mathrm{h}$ of phlebotomy, and the aliquoted plasma samples were stored at $-80^{\circ} \mathrm{C}$ until assay. The study was approved by Institutional Review Boards of the University of Miami and Jackson Memorial Hospital, and all patients provided written informed consent.

The inclusion criteria were adult $(\geq 18$ years old at the time of diagnosis) female patients, newly diagnosed with breast cancer (AJCC stage 0 -III) who had undergone BCS and planned to receive adjuvant RT to the whole breast with or without regional lymph nodes (total dose $\geq 40 \mathrm{~Gy}$, dose per fraction $\geq 2.0 \mathrm{~Gy}$ ). Other criteria included patients belonging to one of three racial/ethnic groups [self-reported non-Hispanic whites (NHW), black/African Americans (AA), and Hispanic whites (HW)] and being able to speak English or Spanish. The exclusion criteria were patients diagnosed with stage IV breast cancer and those that received partial breast irradiation and/or concurrent chemoradiation. Patients with missing pain score and/or CRP level at pre- or post-RT were excluded. To increase the validity of RT-related change in pain score, patients who reported pain due to other acute health conditions unrelated to cancer or radiation (such as shingles or fracture) were also excluded from the analysis after medical record verification.

\section{Radiation treatment}

RT was delivered using standard or partially wide photon tangents using 6 and/or 10MV photons with forward planned field-in-field technique to maximize dose homogeneity. Patients received RT to the whole breast \pm regional lymph nodes with conventional fractionation $(2.0 \mathrm{~Gy} /$ day over 5-6 weeks, mostly 50 Gy in 25 fractions) or hypo-fractionation (>2.0 Gy/day over 3 weeks, most commonly 42.4 Gy in 16 fractions). An additional boost dose of 10-20 Gy without bolus was delivered to the tumor-bed site in most patients. Radiation oncologists contoured target volumes, including the breast and lumpectomy cavity. The treatment plan was completed on the Eclipse or Pinnacle planning systems.

\section{Assessment of pain}

All women enrolled in the study filled out the National Surgical Adjuvant Breast and Bowel Project (NSABP) B-39/ RTOG 0413 protocol QOL questionnaire pre- and post-RT. This questionnaire measured QOL relating to breast cosmesis, fatigue, treatment-related symptoms, and 
perceived convenience of care. The section pertaining to treatment-related symptoms included four pain severity items, which were extracted from the Brief Pain Inventory (BPI): "Rate your pain at its worst, at its least, on average in the past four weeks, and now $(0=$ no pain to $10=$ pain as bad as you can imagine)." The pain score was measured as a mean of these four pain severity items; a pain score of 410 was used to define the presence of clinically relevant pain because pain $\geq 4$ indicates a moderate to severe level of pain, as used in previous studies [7, 24, 25]. In addition, patients who reported an increase in pain level from pre- to post-RT (i.e., pain score changed from $<4$ to $\geq 4$ ) was defined as having RT-related pain as previously reported [11] and compared to patients with pain score $<4$ at both preand post-RT.

\section{Assessment of plasma CRP}

Plasma CRP levels were measured using a high-sensitivity CRP enzyme-linked immunosorbent assay (ELISA) kit (Calbiotech, Spring Valley, CA) according to the manufacturer's protocol, as previously described [16]. A standard curve was generated for each batch of samples based on CRP concentrations, which ranged from 0.2 to $10.0 \mathrm{mg} / \mathrm{L}$. To ensure that the diluted samples were within the linear range of the standard curve, we re-ran the assays by adjusting the dilution ratio if samples were outside the detection range. The average coefficient of variation was $8.3 \%$, and the inter-assay variation was less than $10 \%$. The cut-off value of CRP level was determined based on clinical usage and literature review where $\mathrm{CRP} \geq 10.0 \mathrm{mg} / \mathrm{L}$ is a prognostic biomarker for breast cancer survival [26]. For CRP change, we used $1.0 \mathrm{mg} / \mathrm{L}$ as the cut-off value because it has been significantly associated with RT-induced skin toxicity in the same patient population [16]. Considering that CRP is an acute-phase protein with a half-life of $18 \mathrm{~h}$, we collected post-RT blood samples immediately after RT on the last day consistently among all sample patients.

\section{Assessment of covariates}

Demographic information, self-reported race and ethnicity, comorbidities, and smoking history/status were obtained from a self-administered baseline questionnaire at the time of enrollment. A high correlation was found between the comorbidities reported on the questionnaires and those extracted from medical records [27, 28]. Tumor characteristics, such as tumor stage, estrogen receptor (ER), progesterone receptor (PR), human epidermal growth factor receptor 2 (HER2), and detailed information on treatments were ascertained from medical records.

\section{Statistical analysis}

We first examined the distributions and frequencies of patient-, tumor-, and treatment-related characteristics overall and by race/ethnicity using the Pearson's chi-square test or the Fisher's exact test. The analysis of variance (ANOVA) was used to compare CRP levels by patient characteristics. The Pearson's chi-square test or the Fisher's exact test was used to compare the frequencies of elevated CRP or pain by patient characteristics. Univariable and multivariable logistic regression analyses were used to test whether elevated pre-RT CRP and/or obesity (BMI $\geq 30 \mathrm{~kg} / \mathrm{m}^{2}$ ) were significantly associated with RT-related pain. Odds ratios (ORs) and 95\% confidence intervals (95\% CIs) were reported. In addition, we performed the receiver operating characteristics (ROC) curve analysis to evaluate whether pre-RT CRP level and/or obesity contribute to RT-related pain. A two-tailed $P$ value $<0.05$ was considered statistically significant, and all statistical analyses were performed using SAS 9.4 (SAS Institute, Cary, NC, USA).

\section{Results}

\section{Patient population characteristics}

The study population consisted of 366 breast cancer patients: $64 \% \mathrm{HW}, 20 \% \mathrm{AA}$, and $16 \% \mathrm{NHW}$. The mean \pm standard deviation (SD) of age was $56.0 \pm 9.1$ years. As shown in Table 1, AA women were more likely to have BMI $\geq 30 \mathrm{~kg} / \mathrm{m}^{2}$, advanced stage or triple-negative tumors, larger volume (cc) of the breast, diabetes mellitus, and hypertension compared to HW or NHW women. HW women were more likely to receive hormone therapy (HT) with aromatase inhibitors prior to RT compared to other racial/ethnic groups. For breast cancer surgery, $68 \%$ of patients received BCS with or without sentinel lymph node biopsy (SLNB), and 32\% received BCS with axillary lymph node dissection (ALND). For systemic therapy, about half of the patients received chemotherapy, $44 \%$ initiated HT prior to RT, and 7\% began HT during RT. For RT, $84 \%$ of patients received conventional fractionation with a mean total dose of $58.2 \pm 4.8$ (SD) Gy, including an additional boost to the lumpectomy cavity, and $16 \%$ were treated with hypo-fractionated regimens. There were no significant differences in RT treatment regimens across the three racial/ ethnic groups. Overall, patients reported a significantly higher pain score at post-RT (mean $\pm \mathrm{SD}=2.8 \pm 2.5)$ compared to pre-RT (mean $\pm \mathrm{SD}=1.7 \pm 2.1$ ). In general, $\mathrm{AA}$ and HW patients had significantly higher pre-RT and post-RT pain scores compared to NHW patients.

\section{Plasma CRP levels at pre- and post-RT and RT-related CRP change}

As shown in Table 2, there was no significant difference between pre- (mean $\pm \mathrm{SD}=6.5 \pm 9.3$ ) and post-RT (mean \pm $\mathrm{SD}=6.1 \pm 8.9$ ) plasma CRP levels. The CRP levels were significantly higher in obese patients at both pre- and post-RT. Pre-RT CRP levels were significantly higher in patients with pre- or post-RT pain score $\geq 4$. Post-RT CRP levels were significantly higher in patients with smoking 
Table 1 Patient demographic, tumor, and treatment characteristics by race/ethnicity

\begin{tabular}{|c|c|c|c|c|c|c|c|c|c|c|}
\hline \multirow[t]{2}{*}{ Variable } & \multirow[t]{2}{*}{ Categories } & \multicolumn{2}{|c|}{ Total } & \multicolumn{2}{|c|}{ NHW } & \multicolumn{2}{|c|}{ AA } & \multicolumn{2}{|l|}{ HW } & \multirow[t]{2}{*}{$P^{1}$} \\
\hline & & $N$ & $\%$ & $N$ & $\%$ & $N$ & $\%$ & $N$ & $\%$ & \\
\hline Total & & 366 & 100 & 58 & 16 & 73 & 20 & 235 & 64 & \\
\hline \multirow[t]{3}{*}{ Age (years) } & $<50$ & 95 & 26 & 18 & 31 & 19 & 26 & 58 & 25 & 0.613 \\
\hline & $\geq 50$ & 271 & 74 & 40 & 69 & 54 & 74 & 177 & 75 & \\
\hline & Mean (SD) & \multicolumn{2}{|c|}{$56.0(9.1)$} & \multicolumn{2}{|c|}{$55.6(9.1)$} & \multicolumn{2}{|c|}{$54.9(9.2)$} & \multicolumn{2}{|c|}{$56.5(9.1)$} & \\
\hline \multirow[t]{4}{*}{ BMI $\left(\mathrm{kg} / \mathrm{m}^{2}\right)$} & $<25$ & 96 & 26 & 29 & 50 & 12 & 16 & 55 & 23 & $<0.0001$ \\
\hline & $25-29.9$ & 124 & 34 & 16 & 28 & 17 & 23 & 91 & 39 & \\
\hline & $\geq 30$ & 146 & 40 & 13 & 22 & 44 & 60 & 89 & 38 & \\
\hline & Mean (SD) & \multicolumn{2}{|c|}{$29.3(6.4)$} & \multicolumn{2}{|c|}{$26.6(6.3)$} & \multicolumn{2}{|c|}{$32.6(8.4)$} & \multicolumn{2}{|c|}{$28.9(5.2)$} & \\
\hline \multirow[t]{3}{*}{ Smoking status } & Never & 240 & 66 & 37 & 64 & 51 & 70 & 152 & 64 & 0.490 \\
\hline & Former & 107 & 29 & 20 & 34 & 17 & 23 & 70 & 30 & \\
\hline & Current & 19 & 5 & 1 & 2 & 5 & 7 & 13 & 6 & \\
\hline \multirow[t]{4}{*}{ Sum of 12 comorbid conditions ${ }^{2}$} & 0 & 147 & 40 & 28 & 48 & 19 & 26 & 100 & 43 & 0.119 \\
\hline & 1 & 137 & 37 & 20 & 34 & 32 & 44 & 85 & 36 & \\
\hline & 2 & 60 & 16 & 7 & 12 & 18 & 25 & 35 & 15 & \\
\hline & $\geq 3$ & 22 & 6 & 3 & 5 & 4 & 5 & 15 & 6 & \\
\hline \multirow[t]{4}{*}{ Tumor stage } & 0 & 74 & 20 & 7 & 12 & 14 & 19 & 53 & 23 & 0.003 \\
\hline & $\mid A-B$ & 180 & 49 & 37 & 64 & 28 & 38 & 115 & 49 & \\
\hline & $\| A-B$ & 90 & 25 & 13 & 22 & 29 & 40 & 48 & 20 & \\
\hline & $\| \mathrm{A}-\mathrm{C}$ & 22 & 6 & 1 & 2 & 2 & 3 & 19 & 8 & \\
\hline \multirow[t]{2}{*}{ ER } & Positive & 279 & 76 & 43 & 74 & 49 & 67 & 187 & 80 & 0.072 \\
\hline & Negative & 86 & 23 & 15 & 26 & 24 & 33 & 47 & 20 & \\
\hline PR & Positive & 243 & 66 & 36 & 62 & 44 & 60 & 163 & 69 & 0.243 \\
\hline & Negative & 122 & 33 & 22 & 38 & 29 & 40 & 71 & 30 & \\
\hline HER2 & Positive & 31 & 8 & 4 & 7 & 6 & 8 & 21 & 9 & 0.730 \\
\hline & Negative & 275 & 75 & 50 & 86 & 56 & 77 & 169 & 72 & \\
\hline Triple negative & No & 294 & 80 & 47 & 81 & 52 & 71 & 195 & 83 & 0.005 \\
\hline & Yes & 54 & 15 & 8 & 14 & 20 & 27 & 26 & 11 & \\
\hline Axillary surgery & None/SLNB & 248 & 68 & 39 & 67 & 54 & 74 & 155 & 66 & 0.439 \\
\hline & ALND & 118 & 32 & 19 & 33 & 19 & 26 & 80 & 34 & \\
\hline Chemotherapy & No & 195 & 53 & 31 & 53 & 39 & 53 & 125 & 53 & 0.999 \\
\hline & Yes & 171 & 47 & 27 & 47 & 34 & 47 & 110 & 47 & \\
\hline Hormone therapy/initiation time & None/after RT & 178 & 49 & 37 & 64 & 41 & 56 & 100 & 43 & 0.015 \\
\hline & Aromatase inhibitor before RT & 98 & 27 & 9 & 16 & 14 & 19 & 75 & 32 & \\
\hline & Aromatase inhibitor during RT & 14 & 4 & 3 & 5 & 2 & 3 & 9 & 4 & \\
\hline & Tamoxifen before RT & 64 & 17 & 6 & 10 & 12 & 16 & 46 & 20 & \\
\hline & Tamoxifen during RT & 12 & 3 & 3 & 5 & 4 & 5 & 5 & 2 & \\
\hline RT fractionation & Conventional & 306 & 84 & 45 & 78 & 64 & 88 & 197 & 84 & 0.298 \\
\hline & Нypo & 60 & 16 & 13 & 22 & 9 & 12 & 38 & 16 & \\
\hline Total RT dose (Gy) & $<60$ & 107 & 29 & 21 & 36 & 18 & 25 & 68 & 29 & 0.348 \\
\hline & $\geq 60$ & 259 & 71 & 37 & 64 & 55 & 75 & 167 & 71 & \\
\hline & Mean (SD) & 58.2 & & 58. & & 58 & & 58.0 & & \\
\hline Boost & Yes & 331 & 90 & 56 & 97 & 65 & 89 & 210 & 89 & 0.225 \\
\hline & No & 35 & 10 & 2 & 3 & 8 & 11 & 25 & 11 & \\
\hline
\end{tabular}


Table 1 Patient demographic, tumor, and treatment characteristics by race/ethnicity (Continued)

\begin{tabular}{|c|c|c|c|c|c|c|c|c|c|c|}
\hline \multirow[t]{2}{*}{ Variable } & \multirow[t]{2}{*}{ Categories } & \multicolumn{2}{|c|}{ Total } & \multicolumn{2}{|c|}{ NHW } & \multicolumn{2}{|l|}{ AA } & \multicolumn{2}{|l|}{ HW } & \multirow[t]{2}{*}{$P^{1}$} \\
\hline & & $N$ & $\%$ & $N$ & $\%$ & $N$ & $\%$ & $N$ & $\%$ & \\
\hline \multirow[t]{3}{*}{ Breast volume (cc) } & $<892.1$ (median) & 183 & 50 & 38 & 66 & 20 & 27 & 125 & 53 & $<0.001$ \\
\hline & $\geq 892.1$ (median) & 179 & 49 & 20 & 34 & 52 & 71 & 107 & 46 & \\
\hline & Mean (SD) & \multicolumn{2}{|c|}{996 (532) } & \multicolumn{2}{|c|}{799 (464) } & \multicolumn{2}{|c|}{$1254(645)$} & \multicolumn{2}{|c|}{965 (479) } & \\
\hline Pre-RT pain & Mean (SD) & \multicolumn{2}{|c|}{$1.7(2.1)$} & \multicolumn{2}{|c|}{$1.0(1.3)$} & \multicolumn{2}{|c|}{$2.0(2.5)$} & \multicolumn{2}{|c|}{$1.8(2.1)$} & 0.023 \\
\hline Post-RT pain & Mean (SD) & \multicolumn{2}{|c|}{$2.8(2.5)$} & \multicolumn{2}{|c|}{$1.9(1.7)$} & \multicolumn{2}{|c|}{$3.2(2.6)$} & \multicolumn{2}{|c|}{$2.8(2.6)$} & 0.013 \\
\hline
\end{tabular}

${ }^{1} P$ values from the chi-square test or Fisher's exact test, or ANOVA, excluding missing. Significant findings are in italics

${ }^{2}$ Sum of 12 patient-reported comorbid conditions: diabetes, hypertension, heart disease, lung disease, thyroid disease, cirrhosis liver, stroke, chronic bronchitis, hepatitis, tuberculosis, and 2 others

Abbreviations: NHW non-Hispanic whites, $A A$ black or African American, HW Hispanic whites, SD standard deviation, $B M I$ body mass index, ER estrogen receptor, $P R$ progesterone receptor, HER2 human epidermal growth factor receptor 2, SLNB sentinel lymph node biopsy, ALND axillary lymph node dissection, $R T$ radiotherapy

history, post-RT pain score $\geq 4$, larger breast volume, and tamoxifen treatment during RT.

Clinically relevant pain by selected variables and CRP levels As shown in Table 3, the proportion of patients who reported clinically relevant pain (pain score $\geq 4$ ) increased from $17 \%$ at pre-RT to $30 \%$ at post-RT. Pre-RT pain was more prevalent in patients with AA or HW race/ethnicity, $\mathrm{BMI} \geq 30 \mathrm{~kg} / \mathrm{m}^{2}$, HER2-positive tumor, received trastuzumab alone or taxane+trastuzumab, received ALND, or pre-RT CRP $\geq 10 \mathrm{mg} / \mathrm{L}$, compared to their respective comparison groups. Post-RT pain was more prevalent in patients with AA or HW race/ethnicity, age < 50 years, $\mathrm{BMI} \geq 30 \mathrm{~kg} / \mathrm{m}^{2}$, at least 2 comorbid conditions, conventional RT fractionation, total RT dose $\geq 60 \mathrm{~Gy}$, or pre-RT $\mathrm{CRP} \geq 10 \mathrm{mg} / \mathrm{L}$, compared to their respective counterparts. About 23\% of patients had RT-related pain, and it was more frequent in patients with AA or HW race/ethnicity, at least 2 comorbid conditions, conventional RT fractionation, or RT-induced CRP change $>1 \mathrm{mg} / \mathrm{L}$.

\section{Plasma CRP levels by pain status}

In Table 4 , we summarize CRP levels in 4 or 8 groups of patients and identified significantly higher CRP levels (mean $\pm \mathrm{SD}=10.8 \pm 12.1$ ) in 34 patients with pain scores $\geq$ 4 at both pre- and post-RT. We have also identified 20 patients with pain score $\geq 4$ at pre-RT but $<4$ at post-RT. In stratified analysis by obesity, we identified 11 non-obese patients with high pre-RT CRP also had pain scores $\geq 4$ at both pre- and post-RT. Therefore, we limited subsequent data analysis of RT-related pain to only two groups of patients with pre-RT pain score $<4$ and post-RT score either $<4$ (no) or $\geq 4$ (yes).

\section{Association between pre-RT CRP and RT-related pain}

In Table 5, we evaluated the association of elevated pre-RT CRP ( $\geq 10 \mathrm{mg} / \mathrm{L}$ ) and/or obesity with RT-related pain. In multivariable model, there was a significant association between high pre-RT CRP and RT-related pain $(\mathrm{OR}=2.44,95 \% \mathrm{CI}=1.02,5.85)$ regardless of obesity status. In obese patients, there was a stronger association between high pre-RT CRP and RT-related pain $(\mathrm{OR}=3.71,95 \% \mathrm{CI}=1.05,13.09)$ than in non-obese patients $(\mathrm{OR}=1.36,95 \% \mathrm{CI}=0.35,5.39)$. Therefore, we conducted a combined analysis to show that patients with $\mathrm{BMI} \geq 30 \mathrm{~kg} / \mathrm{m}^{2}$ and pre-RT CRP $\geq 10 \mathrm{mg} / \mathrm{L}$ had 4.73-fold elevated risk for RT-related pain $(95 \% \mathrm{CI}=$ $1.41,15.81)$ compared to patients with $\mathrm{BMI}<30 \mathrm{~kg} / \mathrm{m}^{2}$ and pre-RT CRP $<10 \mathrm{mg} / \mathrm{L}$. All models were adjusted for age and race/ethnicity.

We also present ROC curves of high pre-RT CRP and/ or obesity in predicting RT-related pain for (A) all, (B) NHW, (C) HW, and (D) AA patients and their corresponding area under the curve (AUC). The gray line represents the theoretical performance of the variable equivalent to a coin toss. The blue line is for obesity $\left(\mathrm{BMI} \geq 30 \mathrm{~kg} / \mathrm{m}^{2}\right)$, the red line is for pre-RT CRP $\geq 10 \mathrm{mg} /$ $\mathrm{L}$, and the green line shows the combined effect of obesity and pre-RT CRP $\geq 10 \mathrm{mg} / \mathrm{L}$. The results show some improvements of AUC in the combined BMI and pre-RT CRP model for NHW $(\mathrm{AUC}=0.6540)$ and $\mathrm{AA}(\mathrm{AUC}=$ 0.6524 ) patients (see Additional file 1: Figure S1).

\section{Discussion}

Postoperative adjuvant RT significantly reduces local-regional recurrence and improves breast cancer survival. Therefore, there has been increasing usage of adjuvant RT in early-stage breast cancer patients. However, RT is associated with skin toxicities and other late effects that negatively impact QOL. We evaluated whether the inflammatory biomarker, CRP, was associated with RT-related pain. To the best of our knowledge, this is the first study to date reporting a significant association between pre-RT CRP and RT-related pain.

Consistent with literature, the proportion of patients who experienced clinically relevant pain increased from pre-RT $(17 \%)$ to post-RT $(30 \%)$ [7, 29]. Pre-RT pain may be related to other cancer treatments (e.g., surgery and/ or chemotherapy). Intriguingly, a higher proportion of patients with at least two comorbid conditions showed 
Table 2 CRP levels by patient, treatment characteristics, and pain status

\begin{tabular}{|c|c|c|c|c|c|c|c|c|c|c|}
\hline \multirow[t]{2}{*}{ Variable } & \multicolumn{5}{|c|}{ Pre-RT CRP (mg/L) } & \multicolumn{5}{|c|}{ Post-RT CRP (mg/L) } \\
\hline & $\bar{N}$ & Mean & SD & $M D$ & $P^{1}$ & $\bar{N}$ & Mean & SD & MD & $P^{1}$ \\
\hline Study population & 362 & 6.5 & 9.3 & 3.5 & & 338 & 6.1 & 8.9 & 3.5 & $0.646^{2}$ \\
\hline \multicolumn{11}{|l|}{ Race/ethnicity } \\
\hline NHW & 58 & 6.1 & 12.4 & 2.8 & 0.879 & 53 & 5.2 & 12.1 & 2.2 & 0.541 \\
\hline AA & 71 & 6.9 & 8.0 & 4.4 & & 66 & 7.0 & 6.7 & 5.5 & \\
\hline HW & 233 & 6.4 & 8.8 & 3.5 & & 219 & 6.0 & 8.6 & 3.7 & \\
\hline \multicolumn{11}{|l|}{ Age (years) } \\
\hline$<50$ & 94 & 6.2 & 10.7 & 3.1 & 0.797 & 86 & 5.6 & 10.1 & 2.8 & 0.571 \\
\hline$\geq 50$ & 268 & 6.5 & 8.8 & 3.7 & & 252 & 6.2 & 8.5 & 3.9 & \\
\hline \multicolumn{11}{|l|}{ BMI $\left(\mathrm{kg} / \mathrm{m}^{2}\right)$} \\
\hline$<25$ & 94 & 3.1 & 6.3 & 1.2 & 0.0001 & 87 & 3.1 & 8.4 & 1.4 & 0.0009 \\
\hline $25-29.99$ & 124 & 7.3 & 11.0 & 3.7 & & 117 & 6.5 & 9.5 & 3.8 & \\
\hline$\geq 30$ & 144 & 8.0 & 8.8 & 4.9 & & 134 & 7.7 & 8.4 & 5.3 & \\
\hline \multicolumn{11}{|l|}{ Smoking history } \\
\hline Never & 238 & 5.8 & 8.6 & 3.4 & 0.066 & 225 & 5.4 & 7.8 & 3.4 & 0.046 \\
\hline Ever & 124 & 7.7 & 10.3 & 3.7 & & 113 & 7.4 & 10.7 & 3.9 & \\
\hline \multicolumn{11}{|l|}{ Pre-RT pain score } \\
\hline$<4$ & 286 & 5.9 & 8.8 & 3.3 & 0.014 & 266 & 5.6 & 8.4 & 3.4 & 0.054 \\
\hline$\geq 4$ & 59 & 9.3 & 11.8 & 4.9 & & 56 & 8.1 & 10.3 & 4.2 & \\
\hline \multicolumn{11}{|l|}{ Post-RT pain score } \\
\hline$<4$ & 230 & 5.4 & 8.2 & 3.1 & 0.007 & 226 & 5.3 & 7.8 & 3.2 & 0.014 \\
\hline$\geq 4$ & 101 & 8.3 & 9.9 & 4.6 & & 99 & 7.9 & 11.0 & 4.8 & \\
\hline \multicolumn{11}{|l|}{ Tumor stage } \\
\hline 0 & 73 & 6.1 & 8.2 & 3.6 & 0.916 & 65 & 6.3 & 8.3 & 3.4 & 0.872 \\
\hline $\mid A-B$ & 180 & 6.4 & 10.0 & 3.3 & & 165 & 6.3 & 10.8 & 3.4 & \\
\hline$\|A-\| \|$ & 109 & 6.7 & 8.8 & 3.9 & & 108 & 5.7 & 5.7 & 4.2 & \\
\hline \multicolumn{11}{|l|}{ Breast volume (cc) } \\
\hline < 892.1 cc (median) & 181 & 5.2 & 8.2 & 2.6 & 0.011 & 169 & 5.0 & 9.2 & 2.4 & 0.021 \\
\hline$\geq 892.1$ cc (median) & 177 & 7.7 & 10.2 & 4.6 & & 165 & 7.2 & 8.6 & 5.0 & \\
\hline \multicolumn{11}{|l|}{ Hormone therapy } \\
\hline None/after RT & 175 & 6.7 & 10.5 & 3.2 & 0.736 & 159 & 5.7 & 7.9 & 3.3 & 0.032 \\
\hline Al before & 97 & 6.7 & 7.7 & 4.8 & & 95 & 7.2 & 10.0 & 4.6 & \\
\hline Al during & 14 & 4.5 & 4.0 & 3.3 & & 14 & 5.1 & 5.3 & 3.8 & \\
\hline Tamoxifen before & 64 & 5.5 & 8.8 & 2.8 & & 59 & 4.3 & 6.0 & 2.4 & \\
\hline Tamoxifen during & 12 & 8.2 & 9.3 & 5.1 & & 11 & 12.8 & 21.0 & 5.1 & \\
\hline \multicolumn{11}{|l|}{ RT fractionation } \\
\hline Conventional & 302 & 6.4 & 9.1 & 3.5 & 0.632 & 289 & 6.2 & 9.2 & 3.5 & 0.575 \\
\hline Нyро & 60 & 7.0 & 10.2 & 3.5 & & 49 & 5.4 & 7.0 & 3.7 & \\
\hline
\end{tabular}

${ }^{1} P$ values from ANOVA; significant findings are in italics

${ }^{2}$ Paired $t$ test comparing pre- and post-RT CRP

Abbreviations: NHW non-Hispanic whites, AA black or African American, HW Hispanic whites, BMI body mass index, $A l$ aromatase inhibitor, SD standard deviation, MD median

an elevated risk for post-RT pain [30]. It is notable that not all patients reported an increase in pain score after RT. Specifically, 194 patients reported pain score $<4$ at both pre- and post-RT. A total of 57 patients reported the change of pain score from $<4$ at pre-RT to $\geq 4$ at post-RT. Twenty patients reported pain score change 
Table 3 Pre-RT, post-RT, and RT-related pain by selected variables and CRP status

\begin{tabular}{|c|c|c|c|c|c|c|c|c|c|c|c|c|c|c|c|c|}
\hline \multirow[t]{3}{*}{ Variable } & \multirow[t]{3}{*}{ Categories } & \multicolumn{5}{|c|}{ Pre-RT pain ${ }^{1}(N=349)$} & \multicolumn{5}{|c|}{ Post-RT pain ${ }^{1}(N=335)$} & \multicolumn{5}{|c|}{ RT-related pain ${ }^{2}(N=262)$} \\
\hline & & \multicolumn{2}{|c|}{ No $(<4)$} & \multicolumn{2}{|c|}{ Yes $(\geq 4)$} & \multirow[b]{2}{*}{$P^{3}$} & \multicolumn{2}{|c|}{$\mathrm{No}(<4)$} & \multicolumn{2}{|c|}{ Yes $(\geq 4)$} & \multirow[b]{2}{*}{$P^{3}$} & \multicolumn{2}{|l|}{$\overline{\text { No }}$} & \multicolumn{2}{|c|}{ Yes } & \multirow[b]{2}{*}{$P^{3}$} \\
\hline & & $\bar{N}$ & $\%$ & $\bar{N}$ & $\%$ & & $\bar{N}$ & $\%$ & $\bar{N}$ & $\%$ & & $\bar{N}$ & $\%$ & $\bar{N}$ & $\%$ & \\
\hline Total & & 290 & 83 & 59 & 17 & & 233 & 70 & 102 & 30 & & 203 & 77 & 59 & 23 & \\
\hline \multirow[t]{3}{*}{ Race/ethnicity } & $\mathrm{NHW}$ & 53 & 96 & 2 & 4 & 0.016 & 45 & 88 & 6 & 12 & 0.003 & 42 & 89 & 5 & 11 & 0.018 \\
\hline & AA & 58 & 82 & 13 & 18 & & 42 & 60 & 28 & 40 & & 37 & 66 & 19 & 34 & \\
\hline & HW & 179 & 80 & 44 & 20 & & 146 & 68 & 68 & 32 & & 124 & 78 & 35 & 22 & \\
\hline \multirow[t]{2}{*}{ Age (years) } & $<50$ & 75 & 81 & 17 & 19 & 0.639 & 51 & 60 & 34 & 40 & 0.027 & 46 & 69 & 21 & 31 & 0.045 \\
\hline & $\geq 50$ & 215 & 84 & 42 & 16 & & 182 & 73 & 68 & 27 & & 157 & 81 & 38 & 19 & \\
\hline \multirow[t]{3}{*}{ BMI $\left(\mathrm{kg} / \mathrm{m}^{2}\right)$} & $<25$ & 84 & 88 & 11 & 12 & 0.009 & 68 & 81 & 16 & 19 & 0.001 & 63 & 85 & 11 & 15 & 0.075 \\
\hline & 25-29.99 & 101 & 88 & 14 & 12 & & 85 & 74 & 30 & 26 & & 73 & 78 & 20 & 22 & \\
\hline & $\geq 30$ & 105 & 75 & 34 & 25 & & 80 & 59 & 56 & 41 & & 67 & 71 & 28 & 29 & \\
\hline \multirow[t]{4}{*}{ Sum of 12 comorbid conditions ${ }^{4}$} & 0 & 114 & 84 & 22 & 16 & 0.897 & 103 & 75 & 34 & 25 & 0.009 & 87 & 83 & 18 & 17 & 0.009 \\
\hline & 1 & 111 & 83 & 22 & 17 & & 88 & 73 & 33 & 27 & & 79 & 81 & 18 & 19 & \\
\hline & 2 & 48 & 83 & 10 & 17 & & 32 & 57 & 24 & 43 & & 28 & 64 & 16 & 36 & \\
\hline & $\geq 3$ & 17 & 77 & 5 & 23 & & 10 & 48 & 11 & 52 & & 9 & 56 & 7 & 44 & \\
\hline \multirow[t]{2}{*}{ HER2 } & Positive & 18 & 62 & 11 & 38 & 0.004 & 16 & 59 & 11 & 41 & 0.294 & 10 & 71 & 4 & 29 & 0.676 \\
\hline & Negative & 220 & 84 & 42 & 16 & & 177 & 69 & 79 & 31 & & 155 & 76 & 48 & 24 & \\
\hline \multirow[t]{3}{*}{ Chemotherapy } & None & 159 & 85 & 27 & 15 & 0.140 & 128 & 72 & 50 & 28 & 0.455 & 115 & 80 & 29 & 20 & 0.416 \\
\hline & Taxane & 123 & 79 & 32 & 21 & & 100 & 68 & 48 & 32 & & 83 & 75 & 27 & 25 & \\
\hline & Other & 8 & 100 & 0 & 0 & & 5 & 56 & 4 & 44 & & 5 & 63 & 3 & 38 & \\
\hline \multirow[t]{2}{*}{ Trastuzumab } & No & 274 & 85 & 49 & 15 & 0.005 & 218 & 70 & 92 & 30 & 0.281 & 194 & 78 & 55 & 22 & 0.497 \\
\hline & Yes & 16 & 61 & 10 & 39 & & 15 & 60 & 10 & 40 & & 9 & 69 & 4 & 31 & \\
\hline \multirow[t]{3}{*}{ Taxane+trastuzumab } & None/other chemo only & 166 & 86 & 26 & 14 & 0.012 & 132 & 71 & 53 & 29 & 0.558 & 120 & 79 & 31 & 21 & 0.667 \\
\hline & Either & 109 & 82 & 24 & 18 & & 87 & 68 & 40 & 32 & & 74 & 75 & 25 & 25 & \\
\hline & Both & 15 & 62 & 9 & 38 & & 14 & 61 & 9 & 39 & & 9 & 75 & 3 & 25 & \\
\hline Axillary surgery & None/SLNB & 205 & 86 & 33 & 14 & 0.027 & 163 & 72 & 63 & 28 & 0.141 & 145 & 78 & 40 & 22 & 0.590 \\
\hline & ALND & 85 & 77 & 26 & 23 & & 70 & 64 & 39 & 36 & & 58 & 75 & 19 & 25 & \\
\hline RT fractionation & Conventional & 240 & 82 & 52 & 18 & 0.309 & 190 & 67 & 94 & 33 & 0.013 & 165 & 75 & 55 & 25 & 0.028 \\
\hline & Hypo & 50 & 88 & 7 & 12 & & 43 & 84 & 8 & 16 & & 38 & 90 & 4 & 10 & \\
\hline Total RT dose (Gy) & $<60$ & 92 & 89 & 11 & 11 & 0.045 & 74 & 80 & 19 & 20 & 0.014 & 66 & 84 & 13 & 16 & 0.123 \\
\hline & $\geq 60$ & 198 & 80 & 48 & 20 & & 159 & 66 & 83 & 34 & & 137 & 75 & 46 & 25 & \\
\hline Pre-RT CRP (mg/L) & $<10$ & 256 & 85 & 45 & 15 & 0.006 & 210 & 73 & 79 & 27 & 0.001 & 183 & 79 & 48 & 21 & 0.056 \\
\hline & $\geq 10$ & 30 & 68 & 14 & 32 & & 20 & 48 & 22 & 52 & & 17 & 63 & 10 & 37 & \\
\hline Post-RT CRP (mg/L) & $<10$ & 234 & 83 & 47 & 17 & 0.410 & 203 & 71 & 82 & 29 & 0.077 & 175 & 78 & 49 & 22 & 0.373 \\
\hline & $\geq 10$ & 32 & 78 & 9 & 22 & & 23 & 58 & 17 & 43 & & 22 & 71 & 9 & 29 & \\
\hline RT-related CRP change (mg/L) & $\leq 1$ & 192 & 82 & 41 & 18 & 0.992 & 170 & 72 & 67 & 28 & 0.140 & 151 & 82 & 34 & 18 & 0.006 \\
\hline & $>1$ & 70 & 82 & 15 & 18 & & 53 & 63 & 31 & 37 & & 43 & 65 & 23 & 35 & \\
\hline
\end{tabular}

${ }^{1}$ Pain score $\geq 4$ (moderate or severe pain) was considered yes for clinically relevant pain

${ }^{2}$ Patients with pre-RT pain score $<4$ and post-RT pain score $\geq 4$ or $<4$ were considered yes or no for RT-related pain

${ }^{3} P$ values were from the chi-square test or Fisher's exact test excluding missing. Significant findings are in italics

${ }^{4}$ Sum of 12 patient-reported comorbid conditions: diabetes, hypertension, heart disease, lung disease, thyroid disease, cirrhosis liver, stroke, chronic bronchitis, hepatitis, tuberculosis, and 2 others

from $\geq 4$ at pre-RT to $<4$ at post-RT. Thirty-four patients reported pain score $\geq 4$ at both pre- and post-RT. These findings are consistent with another study among breast cancer patients, which reported that cancer pain was not static, but rather could progress or regress [25]. Inter-individual variations in pain may be related to differences in responses to RT, genetic factors, and inflammatory responses. 
Table 4 CRP levels by pre- and post-RT pain stratified by obesity

\begin{tabular}{|c|c|c|c|c|c|c|c|c|c|c|c|c|}
\hline \multirow[t]{2}{*}{ BMI } & \multirow[t]{2}{*}{ Pre-RT pain } & \multirow[t]{2}{*}{ Post RT pain } & \multirow[t]{2}{*}{ N } & \multicolumn{4}{|c|}{ Pre-RT CRP } & \multicolumn{4}{|c|}{ Post-RT CRP } & \multirow[b]{2}{*}{$P^{2}$} \\
\hline & & & & Mean & SD & Median & $P^{1}$ & $\overline{\text { Mean }}$ & SD & Median & $P^{1}$ & \\
\hline NA & No & No & 194 & 5.5 & 8.4 & 3.3 & 0.278 & 5.2 & 7.9 & 3.2 & 0.034 & 0.675 \\
\hline NA & No & Yes & 57 & 7.1 & 8.8 & 3.4 & & 7.2 & 10.8 & 4.8 & & 0.936 \\
\hline NA & Yes & No & 20 & 5.4 & 9.2 & 2.2 & 0.010 & 5.6 & 9.5 & 3.3 & 0.075 & 0.807 \\
\hline NA & Yes & Yes & 34 & 10.8 & 12.1 & 6.0 & & 8.8 & 10.2 & 5.6 & & 0.278 \\
\hline$<30$ & No & No & 130 & 5.1 & 9.2 & 2.6 & 0.786 & 4.4 & 8.1 & 2.0 & 0.169 & 0.423 \\
\hline$<30$ & No & Yes & 31 & 5.4 & 8.1 & 2.7 & & 7.4 & 14.1 & 3.2 & & 0.366 \\
\hline$<30$ & Yes & No & 10 & 4.4 & 7.0 & 2.2 & 0.393 & 3.6 & 2.4 & 3.1 & 0.647 & 0.676 \\
\hline$<30$ & Yes & Yes & 11 & 12.1 & 16.8 & 3.6 & & 7.5 & 11.5 & 4.2 & & 0.304 \\
\hline$\geq 30$ & No & No & 64 & 6.3 & 6.3 & 4.6 & 0.480 & 6.9 & 7.1 & 4.8 & 0.368 & 0.497 \\
\hline$\geq 30$ & No & Yes & 26 & 9.0 & 9.5 & 5.9 & & 6.9 & 4.8 & 5.9 & & 0.259 \\
\hline$\geq 30$ & Yes & No & 10 & 6.4 & 11.3 & 2.7 & 0.022 & 7.7 & 13.3 & 3.5 & 0.142 & 0.114 \\
\hline$\geq 30$ & Yes & Yes & 23 & 10.1 & 9.4 & 6.4 & & 9.4 & 9.7 & 6.9 & & 0.677 \\
\hline
\end{tabular}

${ }^{1}$ Unadjusted $P$ value from the Wilcoxon two-sample test (comparing 2 groups by pain status)

${ }^{2} P$ value from the paired $t$ test within each group (comparing pre-RT and post-RT CRP). Significant findings are in italics

The CRP level in normal human serum ranges from 0.2 to $10 \mathrm{mg} / \mathrm{L} ; 90 \%$ of apparently healthy individuals have CRP levels $<3 \mathrm{mg} / \mathrm{L}$; and only $1 \%$ have levels $\geq 10$ $\mathrm{mg} / \mathrm{L}$. In our study, $13 \%$ and $13 \%$ of patients had pre-RT and post-RT CRP $\geq 10 \mathrm{mg} / \mathrm{L}$, respectively (Table 3 ). Radiation sensitivity is a complex and inherited polygenic trait, with many genes in multiple biological pathways. Genetic studies are warranted to elucidate the contribution of genetic variants in racial/ethnic differences of RT-related pain. In addition, a higher proportion of AA patients were obese (60\%), compared to $22 \%$ of NHW and $38 \%$ of HW patients, respectively. Other studies have also reported that a higher proportion of AA women had elevated inflammatory cytokines including CRP and interleukin (IL)-6, relative to NHW women
[31, 32]. This may explain, in part, why AA patients experience more cancer treatment-related symptoms such as pain, skin toxicity, nausea/vomiting, and depression compared to NHW patients [11,33-35].

Multiple studies have shown that irradiation increases immune/inflammatory responses [12,36], and there is evidence showing a positive correlation between elevated inflammatory cytokines and pain severity in both human [17, 19] and animal studies [37, 38]. In addition to pain, elevated pro-inflammatory cytokines, including CRP, after cancer treatment have been associated with persistent fatigue and sleep disturbances in breast cancer patients [18, 39]. These findings may suggest the existence of a shared etiology in cancer treatment-related symptoms. Given that immune/inflammation underscores cancer treatment-

Table 5 Association between pre-RT CRP and RT-related pain by obesity

\begin{tabular}{|c|c|c|c|c|c|c|c|c|c|}
\hline \multirow[t]{2}{*}{$\overline{\mathrm{BMI}}$} & \multirow[t]{2}{*}{ Pre-RT CRP } & \multirow[t]{2}{*}{$N$} & \multirow[t]{2}{*}{$\%$} & \multicolumn{2}{|c|}{ RT-related pain } & \multicolumn{2}{|l|}{ Univariable } & \multicolumn{2}{|l|}{ Multivariable } \\
\hline & & & & $\bar{N}$ & $\%$ & OR $(95 \% \mathrm{Cl})$ & $P$ & OR $(95 \% \mathrm{Cl})$ & $P$ \\
\hline$<30$ & NA & 161 & 64 & 31 & 54 & Ref & & Ref & \\
\hline$\geq 30$ & NA & 90 & 36 & 26 & 46 & $1.70(0.93,3.11)$ & 0.082 & $1.49(0.80,2.78)$ & 0.211 \\
\hline NA & $<10 \mathrm{mg} / \mathrm{L}$ & 225 & 90 & 47 & 82 & Ref & & Ref & \\
\hline NA & $\geq 10 \mathrm{mg} / \mathrm{L}$ & 26 & 10 & 10 & 18 & $2.37(1.01,5.55)$ & 0.048 & $2.44(1.02,5.85)$ & 0.046 \\
\hline$<30$ & $<10 \mathrm{mg} / \mathrm{L}$ & 148 & 92 & 28 & 90 & Ref & & Ref & \\
\hline$<30$ & $\geq 10 \mathrm{mg} / \mathrm{L}$ & 13 & 8 & 3 & 10 & $1.29(0.33,4.98)$ & 0.716 & $1.36(0.35,5.39)$ & 0.659 \\
\hline$\geq 30$ & $<10 \mathrm{mg} / \mathrm{L}$ & 77 & 86 & 19 & 73 & Ref & & Ref & \\
\hline$\geq 30$ & $\geq 10 \mathrm{mg} / \mathrm{L}$ & 13 & 14 & 7 & 27 & $3.56(1.07,11.91)$ & 0.039 & $3.71(1.05,13.09)$ & 0.041 \\
\hline$<30$ & $<10 \mathrm{mg} / \mathrm{L}$ & 148 & 59 & 28 & 49 & Ref & & Ref & \\
\hline$<30$ & $\geq 10 \mathrm{mg} / \mathrm{L}$ & 13 & 5 & 3 & 5 & $1.29(0.33,4.98)$ & 0.716 & $1.34(0.34,5.26)$ & 0.678 \\
\hline$\geq 30$ & $<10 \mathrm{mg} / \mathrm{L}$ & 77 & 31 & 19 & 33 & $1.40(0.73,2.72)$ & 0.315 & $1.22(0.62,2.42)$ & 0.567 \\
\hline$\geq 30$ & $\geq 10$ mg/L & 13 & 5 & 7 & 12 & $5.00(1.56,16.03)$ & 0.007 & $4.73(1.41,15.81)$ & 0.012 \\
\hline
\end{tabular}

${ }^{1}$ All models were adjusted for age $(<50, \geq 50)$ and race/ethnicity (NHW, HW, AA). Significant findings are in italics 
related symptoms, the use of anti-inflammatory agents as prophylactic treatment may be considered.

Our current data provides evidence that CRP is associated with RT-related pain in breast cancer patients. Our findings have several clinical implications. First, elevated plasma CRP has been associated with cancer prognosis, vascular atherosclerosis, insulin resistance, and type 2 diabetes mellitus that may impact overall survival. Therefore, patients with elevated post-RT CRP levels should be actively monitored for other medical conditions that may also impact overall survival. Second, considering the involvement of CRP in fatigue and prognosis of breast cancer, future follow-up studies will focus on monitoring CRP levels, QOL, and clinical outcomes. Third, growing evidence suggests that plasma CRP is positively associated with sugar intake but negatively associated with dietary intakes of minerals, vitamins, and polyunsaturated fatty acids [40]. Therefore, modulating CRP concentrations by modifying dietary intakes may be a promising intervention strategy. Lastly, we observed a stronger association between elevated pre-RT CRP and RT-related pain in obese patients. Considering that CRP and BMI are highly correlated, weight reduction may also reduce pre-RT CRP levels and RT-related pain.

Multiple studies have shown the predictive value of CRP in cancer outcomes [41-43]. This study further adds to the literature by reporting a significant association between elevated pre-RT CRP level and RT-related pain. However, using a threshold AUC of 0.8 by ROC analysis, combining BMI and pre-RT CRP levels may not be a strong predictor for RT-related pain. With a limited sample size, we did not include many other clinical or treatment variables. Larger studies are warranted to further test our predictive models, which should include other patient/clinical variables and additional promising biomarkers to improve their utilities in predicting RT-related pain.

There are several strengths and limitations of this study. First, we used a prospective study design that is particularly suitable to conduct biomarker research and RT-related pain. We followed patients and collected biological samples over time and recorded patient-reported QOL on the first and last day of RT to minimize recall bias, which provides more precise estimates of biomarkers and pain. This is the first study showing racial/ethnic differences in pre- and post-RT pain, which may help bridge the knowledge gap regarding the mechanisms of racial/ ethnic disparities in cancer treatment-related QOL.

Several limitations should also be taken into consideration. First, because CRP is a non-specific inflammatory biomarker, CRP levels can be influenced by multiple factors including anti-inflammatory drug use and/or other health conditions. Second, despite the prospective cohort study design, some covariates (i.e., comorbidities) were collected only one point in time. The lack of repeated measures prevented us from capturing changes in health status, which may influence CRP and pain levels. Third, some variables that may influence individual patient's pain experience and CRP level (i.e., the use of pain medication and anti-inflammatory agents) were not available for this study, thus should be considered for future studies. Fourth, the nature of pain (nociceptive or neuropathic) may be differently influenced by inflammatory responses; however, the detailed pain quality data was not available in the current analysis. Lastly, we used patient-reported information on comorbid conditions, which might introduce reporting bias. However, many studies have reported high reliability of self-reported information when compared to medical records [27, 28].

\section{Conclusions}

In summary, our current data show a significant association between elevated pre-RT CRP and RT-related pain in breast cancer patients. More importantly, we demonstrate for the first time that obese patients with pre-RT CRP $\geq 10$ $\mathrm{mg} / \mathrm{L}$ have a significantly increased risk of RT-related pain compared to non-obese patients with pre-RT CRP $<10$ $\mathrm{mg} / \mathrm{L}$. Therefore, our current data suggest that there is an association between inflammatory responses and RT-related pain. Our results will need to be validated externally in other study populations. If validated, these results pave the way for testing anti-inflammatory agents in reducing RT-related pain.

\section{Additional file}

Additional file 1: Figure S1. ROC curves analysis of high pre-RT CRP and/or obesity in RT-related pain. (A) All, (B) NHW, (C) HW, and (D) AA patients and their corresponding AUC for RT-related pain. The grey solid line represents the theoretical performance of the variable equivalent to a coin toss. The blue line represents obesity (BMI $\geq 30$ ), the red line represents pre-RT CRP $\geq 10 \mathrm{mg} / \mathrm{L}$, and the green line presents the combined effect of obesity and pre-RT CRP $\geq 10 \mathrm{mg} / \mathrm{L}$. (PDF $57 \mathrm{~kb}$ )

\section{Abbreviations \\ AA: African American/black; ALND: Axillary lymph node dissection; BCS: Breast-conserving surgery; BMI: Body mass index; Cl: Confidence interval; CRP: C-reactive protein; ER: Estrogen receptor; HER2: Human epidermal growth factor receptor 2; HT: Hormone therapy; HW: Hispanic whites; NHW: Non-Hispanic whites; OR: Odds ratio; PR: Progesterone receptor; QOL: Quality of life; RT: Radiotherapy; SD: Standard deviation; SLNB: Sentinel lymph node biopsy}

\section{Acknowledgements}

The authors are thankful to all women who participated in the study and the clinical staff at the radiation oncology clinics for their support.

\section{Funding}

This study was supported by two National Institutes of Health grants R01CA135288 and R03CA195643 (J.H.) and the University of Miami Sheila and David Fuente Neuropathic Pain Pre-Doctoral fellowship (E.L). 


\section{Availability of data and materials}

The datasets used and/or analyzed during the current study are available from the corresponding author on reasonable request.

\section{Authors' contributions}

$E L, C T$, JLW, IMR, and JJH designed the study. CT and JLW were in charge of radiotherapy, patient enrollment, and clinical outcome assessment. EL, ON, $\mathrm{CP}, \mathrm{CT}, \mathrm{JLW}$, and $\mathrm{JJH}$ collected the laboratory and questionnaire data. EL, RL, $I R$, and WZ conducted the statistical data analysis, and EL and JH interpreted results. EL and JJH drafted the manuscript. All authors read and approved the final manuscript.

\section{Ethics approval and consent to participate}

All women participated in the study provided written informed consent. The study was approved by the Institutional Review Boards of the University of Miami and the Jackson Memorial Hospital.

\section{Consent for publication}

Not applicable.

\section{Competing interests}

The authors declare that they have no competing interests.

\section{Publisher's Note}

Springer Nature remains neutral with regard to jurisdictional claims in published maps and institutional affiliations.

\section{Author details}

'Department of Health Sciences, University of Central Florida College of Health Professions and Sciences, Orlando, FL 32816, USA. ${ }^{2}$ Department of Public Health Sciences, University of Miami School of Medicine, Miami, FL 33136, USA. ${ }^{3}$ Department of Radiation Oncology, University of Miami School of Medicine, Miami, FL, USA. ${ }^{4}$ Department of Radiation Oncology and Molecular Radiation Sciences, Johns Hopkins University, Baltimore, MD, USA. ${ }^{5}$ Sylvester Comprehensive Cancer Center, University of Miami School of Medicine, Miami, FL, USA.

\section{Received: 18 December 2018 Accepted: 6 May 2019}

\section{Published online: 28 May 2019}

\section{References}

1. Siegel RL, Miller KD, Jemal A. Cancer statistics, 2018. CA Cancer J Clin. 2018; 68(1):7-30.

2. Early Breast Cancer Trialists' Collaborative G, Darby S, McGale P, Correa C, Taylor C, Arriagada R, Clarke M, Cutter D, Davies C, Ewertz M, et al. Effect of radiotherapy after breast-conserving surgery on 10-year recurrence and 15year breast cancer death: meta-analysis of individual patient data for 10,801 women in 17 randomised trials. Lancet. 2011;378(9804):1707-16.

3. Pignol JP, Olivotto I, Rakovitch E, Gardner S, Sixel K, Beckham W, Vu TT, Truong P, Ackerman I, Paszat L. A multicenter randomized trial of breast intensity-modulated radiation therapy to reduce acute radiation dermatitis. J Clin Oncol. 2008;26(13):2085-92.

4. Collette S, Collette L, Budiharto T, Horiot JC, Poortmans PM, Struikmans H, Van den Bogaert W, Fourquet A, Jager JJ, Hoogenraad W, et al. Predictors of the risk of fibrosis at 10 years after breast conserving therapy for early breast cancer: a study based on the EORTC trial 22881-10882 'boost versus no boost'. Eur J Cancer. 2008;44(17):2587-99.

5. Poortmans PM, Collette L, Horiot JC, Van den Bogaert WF, Fourquet A, Kuten A, Noordijk EM, Hoogenraad W, Mirimanoff RO, Pierart M, et al. Impact of the boost dose of 10 Gy versus 26 Gy in patients with early stage breast cancer after a microscopically incomplete lumpectomy: 10-year results of the randomised EORTC boost trial. Radiother Oncol. 2009:90(1):80-5.

6. Buchholz TA. Radiation therapy for early-stage breast cancer after breastconserving surgery. N Engl J Med. 2009;360(1):63-70.

7. Gartner R, Jensen MB, Nielsen J, Ewertz M, Kroman N, Kehlet H. Prevalence of and factors associated with persistent pain following breast cancer surgery. J Am Med Assoc. 2009;302(18):1985-92.

8. Whelan TJ, Levine M, Julian J, Kirkbride P, Skingley P. The effects of radiation therapy on quality of life of women with breast carcinoma: results of a randomized trial. Ontario Clinical Oncology Group. Cancer. 2000;88(10):2260-6.
9. Chen SC, Lai YH, Liao CT, Lin CC, Chang JT. Changes of symptoms and depression in oral cavity cancer patients receiving radiation therapy. Oral Oncol. 2010;46(7):509-13.

10. Mak KS, Chen YH, Catalano PJ, Punglia RS, Wong JS, Truong L, Bellon JR. Dosimetric inhomogeneity predicts for long-term breast pain after breastconserving therapy. Int J Radiat Oncol Biol Phys. 2014;93(5):1087-95.

11. Lee E, Takita C, Wright JL, Reis IM, Zhao W, Nelson OL, Hu JJ. Characterization of risk factors for adjuvant radiotherapy-associated pain in a tri-racial/ethnic breast cancer population. Pain. 2016;157(5):1122-31.

12. Mukherjee D, Coates PJ, Lorimore SA, Wright EG. Responses to ionizing radiation mediated by inflammatory mechanisms. J Pathol. 2014;232(3):289-99.

13. Ki Y, Kim W, Nam J, Kim D, Park D, Kim D. C-reactive protein levels and radiation-induced mucositis in patients with head-and-neck cancer. Int J Radiat Oncol Biol Phys. 2009;75(2):393-8.

14. Chen MF, Chen WC, Lai CH, Hung CH, Liu KC, Cheng YH. Predictive factors of radiation-induced skin toxicity in breast cancer patients. BMC Cancer. 2010;10:508

15. Hu JJ, Urbanic JJ, Case LD, Takita C, Wright JL, Brown DR, Langefeld CD, Lively MO, Mitchell SE, Thakrar A, et al. Association between inflammatory biomarker Creactive protein and radiotherapy-induced early adverse skin reactions in a multiracial/ethnic breast cancer population. J Clin Oncol. 2018;36(24):2473-82.

16. Rodriguez-Gil JL, Takita C, Wright J, Reis IM, Zhao W, Lally BE, Hu JJ. Inflammatory biomarker C-reactive protein and radiotherapy-induced early adverse skin reactions in patients with breast cancer. Cancer Epidemiol Biomarkers Prev. 2014;23(9):1873-83.

17. Oliveira KG, von Zeidler SV, Lamas AZ, Podesta JR, Sena A, Souza ED, Lenzi J, Lemos EM, Gouvea SA, Bissoli NS. Relationship of inflammatory markers and pain in patients with head and neck cancer prior to anticancer therapy. Braz J Med Biol Res. 2014;47(7):600-4.

18. Starkweather AR, Lyon DE, Schubert CM. Pain and inflammation in women with early-stage breast cancer prior to induction of chemotherapy. Biol Res Nurs. 2013;15(2):234-41.

19. Laird BJ, Scott AC, Colvin LA, McKeon AL, Murray GD, Fearon KC, Fallon MT. Cancer pain and its relationship to systemic inflammation: an exploratory study. Pain. 2011;152(2):460-3.

20. Archer JA, Hutchison IL, Dorudi S, Stansfeld SA, Korszun A. Interrelationship of depression, stress and inflammation in cancer patients: a preliminary study. J Affect Disord. 2012;143(1-3):39-46.

21. Pertl MM, Hevey D, Boyle NT, Hughes MM, Collier S, O'Dwyer AM, Harkin A, Kennedy MJ, Connor TJ. C-reactive protein predicts fatigue independently of depression in breast cancer patients prior to chemotherapy. Brain Behav Immun. 2013:34:108-19.

22. Miaskowski C, Cooper B, Paul SM, West C, Langford D, Levine JD, Abrams G, Hamolsky D, Dunn L, Dodd M, et al. Identification of patient subgroups and risk factors for persistent breast pain following breast cancer surgery. J Pain. 2012;13(12):1172-87.

23. Langford DJ, Schmidt B, Levine JD, Abrams G, Elboim C, Esserman L, Hamolsky D, Mastick J, Paul SM, Cooper B, et al. Preoperative breast pain predicts persistent breast pain and disability after breast cancer surgery. J Pain Symptom Manag. 2015;49(6):981-94.

24. Sipila R, Estlander AM, Tasmuth T, Kataja M, Kalso E. Development of a screening instrument for risk factors of persistent pain after breast cancer surgery. Br J Cancer. 2012;107(9):1459-66.

25. Mejdahl MK, Andersen KG, Gartner R, Kroman N, Kehlet H. Persistent pain and sensory disturbances after treatment for breast cancer: six year nationwide follow-up study. BMJ. 2013;346:11865.

26. Villasenor A, Flatt SW, Marinac C, Natarajan L, Pierce JP, Patterson RE. Postdiagnosis C-reactive protein and breast cancer survivorship: findings from the WHEL study. Cancer Epidemiol Biomarkers Prev. 2014;23(1):189-99.

27. Ye F, Moon DH, Carpenter WR, et al. Comparison of patient report and medical records of comorbidities: results from a population-based cohort of patients with prostate cancer. JAMA Oncol. 2017;3(8):1035-42.

28. Pit SW, Byles JE, Cockburn J. Accuracy of telephone self-report of drug use in older people and agreement with pharmaceutical claims data. Drugs Aging. 2008;25(1):71-80.

29. Meretoja TJ, Leidenius MH, Tasmuth T, Sipila R, Kalso E. Pain at 12 months after surgery for breast cancer. J Am Med Assoc. 2014;311(1):90-2.

30. Vissers PA, Thong MS, Pouwer F, Zanders MM, Coebergh JW, Van de PollFranse LV. The impact of comorbidity on health-related quality of life among cancer survivors: analyses of data from the PROFILES registry. J Cancer Surviv. 2013;7(4):602-13. 
31. Khera A, McGuire DK, Murphy SA, Stanek HG, Das SR, Vongpatanasin W, Wians FH Jr, Grundy SM, de Lemos JA. Race and gender differences in Creactive protein levels. J Am Coll Cardiol. 2005;46(3):464-9.

32. Park NJ, Kang DH. Inflammatory cytokine levels and breast cancer risk factors: racial differences of healthy caucasian and african american women. Oncol Nurs Forum. 2013;40(5):490-500.

33. Maly RC, Liu Y, Leake B, Thind A, Diamant AL. Treatment-related symptoms among underserved women with breast cancer: the impact of physicianpatient communication. Breast Cancer Res Treat. 2010;119(3):707-16.

34. Martinez KA, Snyder CF, Malin JL, Dy SM. Is race/ethnicity related to the presence or severity of pain in colorectal and lung cancer? J Pain Symptom Manag. 2014;48:1050-9.

35. Wright JL, Takita C, Reis IM, Zhao W, Lee E, Hu JJ. Racial variations in radiationinduced skin toxicity severity: data from a prospective cohort receiving postmastectomy radiation. Int J Radiat Oncol Biol Phys. 2014:90(2):335-43.

36. Hekim N, Cetin Z, Nikitaki Z, Cort A, Saygili El. Radiation triggering immune response and inflammation. Cancer Lett. 2015;368(2):156-63.

37. Lu SG, Gold MS. Inflammation-induced increase in evoked calcium transients in subpopulations of rat dorsal root ganglion neurons. Neuroscience. 2008;153(1):279-88,

38. Moalem-Taylor G, Allbutt HN, lordanova MD, Tracey DJ. Pain hypersensitivity in rats with experimental autoimmune neuritis, an animal model of human inflammatory demyelinating neuropathy. Brain Behav Immun. 2007;21(5):699-710.

39. Collado-Hidalgo A, Bower JE, Ganz PA, Cole SW, Irwin MR. Inflammatory biomarkers for persistent fatigue in breast cancer survivors. Clin Cancer Res. 2006;12(9):2759-66.

40. Mazidi M, Kengne AP, Katsiki N, Mikhailidis DP, Banach M. Inverse association between serum antioxidant levels and inflammatory markers is moderated by adiposity: a report based on a large representative population sample of American adults. Br J Nutr. 2018;120(11):1272-8,

41. Pierce BL, Ballard-Barbash R, Bernstein L, Baumgartner RN, Neuhouser ML, Wener MH, Baumgartner KB, Gilliland FD, Sorensen BE, McTiernan A, et al. Elevated biomarkers of inflammation are associated with reduced survival among breast cancer patients. J Clin Oncol. 2009;27(21):3437-44.

42. Thurner EM, Krenn-Pilko S, Langsenlehner U, Stojakovic T, Pichler M, Gerger A, Kapp KS, Langsenlehner T. The elevated C-reactive protein level is associated with poor prognosis in prostate cancer patients treated with radiotherapy. Eur J Cancer. 2015;51(5):610-9.

43. Basu S, Harris H, Larsson A, Vasson MP, Wolk A. Is there any role for serum cathepsin S and CRP levels on prognostic information in breast cancer? The Swedish mammography cohort. Antioxid Redox Signal. 2015;23(16):1298-302.

Ready to submit your research? Choose BMC and benefit from:

- fast, convenient online submission

- thorough peer review by experienced researchers in your field

- rapid publication on acceptance

- support for research data, including large and complex data types

- gold Open Access which fosters wider collaboration and increased citations

- maximum visibility for your research: over $100 \mathrm{M}$ website views per year

At $\mathrm{BMC}$, research is always in progress.

Learn more biomedcentral.com/submissions 